\title{
Spontaneous regression of cardiac sarcoidosis resulting in total occlusion of coronary artery and ventricular aneurysm
}

\author{
Seok Jong Ryu ${ }^{1}$, Yong Kook Hong ${ }^{1}$, Sanghoon Shin $^{2}$, Sungjun $\mathrm{Cho}^{2}$, and Se-Jung Yoon ${ }^{2}$
}

${ }^{1}$ Department of Radiology, ${ }^{2}$ Division of Cardiology,

Department of Internal Medicine, National Health Insurance Service Ilsan Hospital, Goyang, Korea
Received: August 2, 2016

Revised : August 23, 2016 Accepted: August 23, 2016

\section{Correspondence to}

Se-Jung Yoon, M.D.

Tel: +82-31-900-0630

Fax: +82-31-900-0933

E-mail:drpuooh@hanmail.net
A 68-year-old man with no risk factor of atherosclerosis presented to the cardiology division for ongoing dyspnea on exertion for 1 month. The chest computed tomography (CT) revealed a well-defined huge myocardial mass in the anterior wall of the left ventricle (LV) and multiple pathological lymphadenopathies at the supraclavicular, mediastinal, and right intrapulmonary stations. A diffuse myocardial invasion of a hypoechoic irregular mass was observed in the septum and the anterior portion of the LV $(5.5 \times 3.2 \mathrm{~cm})$ on the transthoracic echocardiography (TTE). The latter showed severe hypokinesia from the mid-LV to the apex combined with a huge myocardial mass and en-
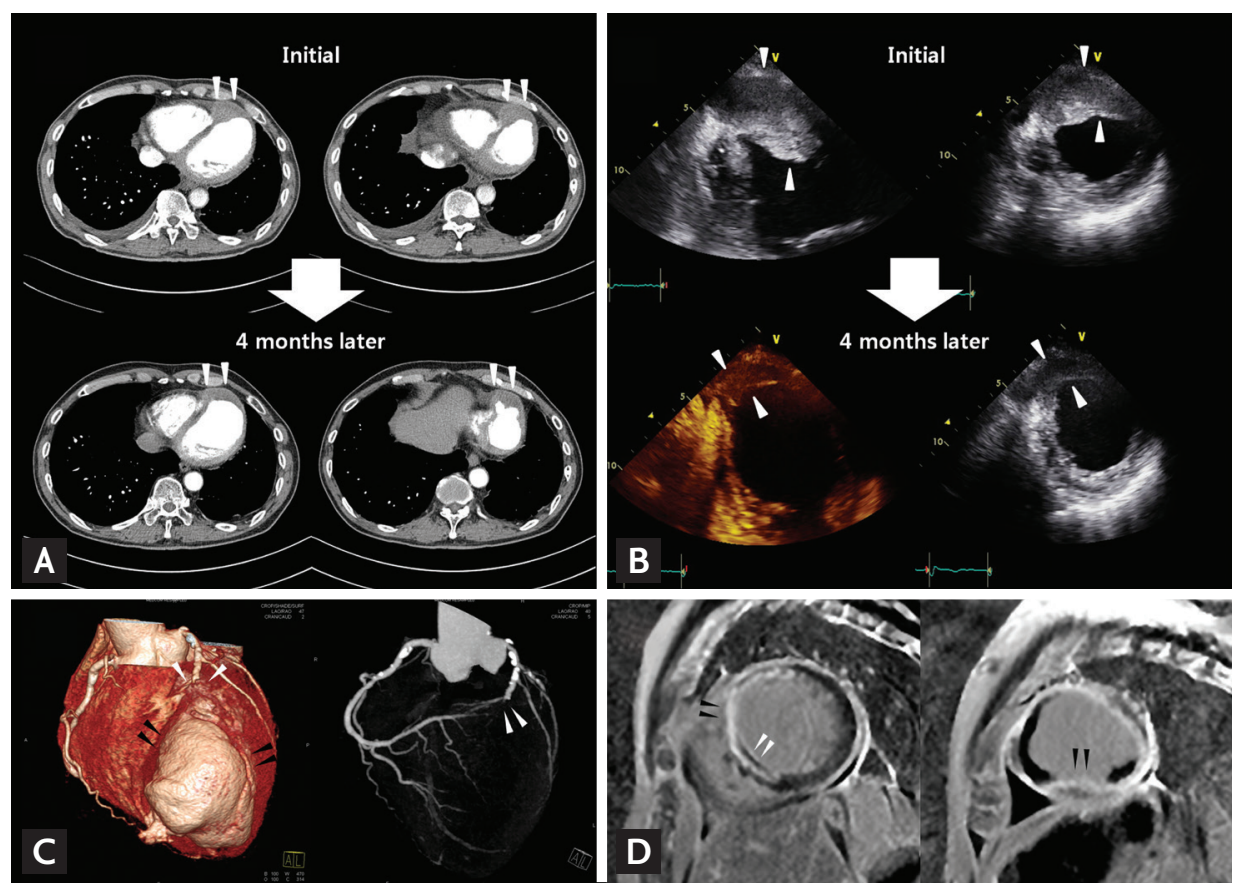

Figure 1. (A, B) Chest computed tomography (CT) and transthoracic echocardiography revealing decreased left ventricle (LV) mass size and newly developed aneurysmal change in apical portion of LV after 4 months (white arrowheads). (C) The coronary CT shows an abruptly interrupted left anterior descending coronary artery (white arrowheads) just before the protruding LV mass and aneurysm (black arrowheads). (D) Cardiac magnetic resonance imaging revealed apical aneurysm and both epicardial and endocardial involvement in delayed hyperenhancement of the septum and the apical portion of the LV (arrowheads). 
larged cardiac chambers.

The excision of the cervical lymph node revealed a granulomatous inflammation suggesting tuberculosis or sarcoidosis. In consideration of the patient's cardiac mass and of his negative tuberculous polymerase chain reaction testing, cardiac sarcoidosis was suspected. The patient refused to undergo a myocardial biopsy or further treatment. After 4 months, a follow-up chest CT and TTE revealed a decrease in the size of the LV mass and a newly developed aneurysmal change in the apical portion of the LV (Fig. $1 \mathrm{~A}$ and $1 \mathrm{~B}$, white arrowheads), with decreased mediastinal and cervical lymph nodes. The coronary CT showed an abrupt interruption of the left anterior descending coronary artery (white arrowheads) just before the protruding
LV mass and aneurysm (black arrowheads) (Fig. 1C). Cardiac magnetic resonance imaging revealed apical aneurysm and both epicardial and endocardial involvement in the delayed hyperenhancement of the septum and the apical portion of the LV (Fig. 1D).

This case was significant in demonstrating that even self-regressing sarcoidosis can cause critical complications such as irreversible myocardial damage or consecutive fatal vascular injury. A multiple imaging approach can facilitate a more accurate and earlier diagnosis.

\section{Conflict of interest}

No potential conflict of interest relevant to this article was reported. 